\title{
Cancer trends in Eastern India: Retrospective hospital-based cancer registry data analysis
}

\author{
Avinash Pandey, Shraddha Raj', Richa Madhawi', Seema Devi', Rajesh Kumar Singh'
}

\begin{abstract}
Background: Trends of cancer cases vary across several hospital-based cancer registries (HBCRs). There is a paucity of demographic data to evaluate trends of cancer in Eastern India. Aim: The aim of this study is to evaluate trends and pattern of cancer cases with respect to time from HBCR from Bihar. Objectives: The objective of this study is to evaluate the numbers of consecutive patients registered with eight most common type of cancer in our HBCR in Regional Cancer Centre, Bihar, and to evaluate trends of cancer cases registered with respect to time. Materials and Methods: Demographic profile of consecutive cancer patients registered from January 2014 to December 2016 (3 years) in HBCR was obtained. Patients diagnosed with common malignancies including head-and-neck cancer, gallbladder, breast, cervix, ovary, esophagus, stomach, hematolymphoid, and colorectal were analyzed. Frequency distribution, crosstabs, and line diagram were used to evaluate the trends of these common cancers with respect to time. Results: Sixty-six thousand and twenty-nine consecutive patients were registered between 2014 and 2016 . Carcinoma gallbladder was the most common malignancy (2I\%), followed by head-and-neck cancer (19\%) and breast cancer (I5\%). Median age at the diagnosis was 55 years for carcinoma gallbladder while 53 years and 46 years for head-and-neck and breast cancer, respectively. Male-to-female ratio was 0.6 for carcinoma gallbladder and 1.8 for head-and-neck cancer.A number of gallbladder and head-and-neck cancer registered increased by $36 \%$ (between 2014 and 20I5) and 5\% (between 2015 and 20I6) and 24\% (between 2014 and 20I5) and 4\% (between 20I5 and 20I6), respectively. Carcinoma breast and cervix showed decreasing trend with fall in registration up to $13 \%$ (between 2015 and 20I6) and $27 \%$ (between 2015 and 2016 ), respectively. Conclusion: Carcinoma gallbladder is the most common cancer in Bihar. Head-and-neck cancer and carcinoma gallbladder are increasing while breast and cervical cancers are decreasing with respect to time.
\end{abstract}

Key words: Bihar, cancer registry, cancer trend

\section{Introduction}

About 14 million new cancer cases and 8 million deaths occur per year globally as international agency for research on cancer report on world cancer statistics. ${ }^{[1]}$ In India, 1.15 million people will be diagnosed with cancer in 2018 with about 0.7 million people dying because of it. ${ }^{[2]}$ Projections estimate that the incidence of cancer is going to further increase to 1.8 million new cases per year by $2026 .{ }^{[3]}$ In Bihar, the burden of new cancer cases would further increase to $1,36,000$ cases in 2026 compared to 86,000 cases in 2011, thus making it fifth most common cancer afflicted state after Uttar Pradesh, Maharashtra, West Bengal, and Andhra Pradesh. ${ }^{[3]}$

As Bihar still lacks population-based cancer registry (PBCR), current data and future predictions are based on crude hospital-based cancer registries (HBCRs) having certain inherent limitations and bias. As per best of knowledge of authors, there is a lack of literature regarding the pattern of incidence of cancers cases in Bihar. We obtained and analyzed data from our HBCR over the past 3 years to learn the pattern of new cancer cases registered in our tertiary regional cancer center.

\section{Materials and Methods}

Our institute is Regional Cancer Center placed in Indira Gandhi Institute of Medical Sciences, Patna, Bihar, where every cancer patient is registered prospectively in HBCR. The demographic profile of each consecutive new patient registered in our HBCR between January 1, 2014, and December 31, 2016, was obtained. Patients were stratified with respect to site and type of cancer along with gender stratification. Cancer types were later analyzed individually and sequentially with respect to descending number of patients registered per site/type of malignancies.

Frequency distribution, crosstabs, and line diagrams were used to evaluate the time trends of the above common malignancies.

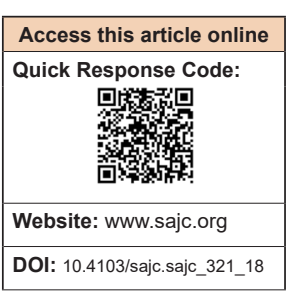

Departments of Medical Oncology and 'Radiotherapy, Indira Gandhi Institute of Medical Sciences, Patna, Bihar, India

Correspondence to: Dr.Avinash Pandey,

E-mail:dr.avinashp9@gmail.com

\section{Results}

Data of consecutive new cancer patients registered between January 1, 2014, and December 31, 2016, in HBCR were retrieved from database along with their baseline demographic profile. During this 3-year tenure, a total of 6629 new cases were registered. The median age of the diagnosis was 49 years across all malignancies.

Overall, most common of all cancers was carcinoma gallbladder $(n=1404)$ across all three consecutive years, with median age at the diagnosis of 55 years and male: female ratio of 0.6 . The second most common was head-and-neck cancer $(n=1275)$ with median age of 53 years at the diagnosis and male: female ratio of 1.8. When stratified across sexes, gallbladder cancer was second most common cancer among females $(n=882)$ after breast cancer, while head-and-neck cancer was most common among males $(n=708)$ closely followed by gallbladder cancer as second $(n=522)$ in the male population. Among females, after breast, gallbladder, and cervical cancer, head-and-neck cancer was forth common cancer $(n=567)$. With respect to time, both gallbladder and head-and-neck cancer showed upward trend with number of gallbladder and head-and-neck cancer registered increased by 36\% (between 2014 and 2015) and 5\% (between 2015 and 2016) and 24\% (between 2014 and 2015) and 4\% (between 2015 and 2016), respectively [Figure 1].

Breast $(n=1018)$ and cervical cancer $(n=713)$ were third and fourth most common cancer. Both demonstrated downward trend with fall in registration by $13 \%$ and $27 \%$ between January 2015 and December 2016, respectively. Median age at the diagnosis for breast and cervical cancer was 46 years and 50 years, respectively [Table 1].

This is an open access journal, and articles are distributed under the terms of the Creative Commons Attribution-NonCommercial-ShareAlike 4.0 License, which allows others to remix, tweak, and build upon the work non-commercially, as long as appropriate credit is given and the new creations are licensed under the identical terms.

For reprints contact: reprints@ medknow.com

How to cite this article: Pandey A, Raj S, Madhawi R, Devi S, Singh RK. Cancer trends in Eastern India: Retrospective hospital-based cancer registry data analysis. South Asian J Cancer 2019;8:215-7. 
Among other cancers, hematolymphoid malignancies $(n=418)$, colorectal cancer $(n=389)$, stomach cancer $(n=324)$, ovarian cancer $(n=291)$, cancer esophagus $(n=170)$, and lung cancer $(n=150)$ were fifth, sixth, seventh, eighth, ninth, and tenth, respectively. Median age at the diagnosis was longest with esophageal cancer, 60 years and shortest in hematological malignancies at 33 years. Among these, colorectal cancer has shown 53\% increase between year 2015 and 2016 while others have nearly plateau to mild increase over the past 3 years.

Beyond these common malignancies, genitourinary $(n=150)$, soft-tissue sarcoma adult $(n=118)$, and pediatric (nonhematolymphoid) malignancies $(n=106)$ were eleventh, twelfth, and thirteenth, respectively. Ninety-six patients had cancers which were not classifiable in the above categories and hence were clubbed together as miscellaneous.

\section{Discussion}

Cancer burden is projected to escalate to 1.8 million new cases by 2026 in India, thus making it the second most common cause of noncommunicable death after cardiovascular diseases. ${ }^{[3]}$ Hence, it is incumbent on government to gear up to provide wider network of diagnostic and therapeutic establishments with more health professionals to meet this impending challenge. The projection of rise in cancer is estimated on the currently available data through PBCRs as part of consortium of the National Cancer Registry Programme. Several major eastern states including most populous Uttar Pradesh, Bihar (third), and West Bengal (forth) till

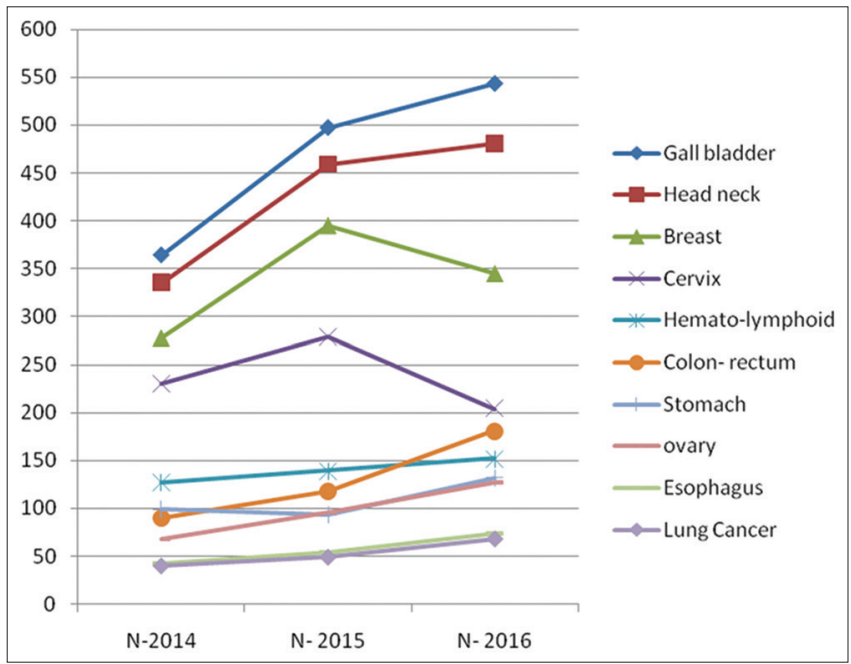

Figure 1: Time trends for ten most common cancers registered between January 2014 and December 2016 in descending orders in Hospital-Based Cancer Registry, Regional Cancer Centre, Patna, Bihar date do not have PBCRs despite having 32\% of Indians living in them. ${ }^{[4,5]}$ This deficit makes cancer projections less reliable in the above states as epidemiological profile of cancer in these states is scarcely known. HBCR is the next best source available by which current cancer incidence trend can be learned. We retrieved and analyzed all consecutive patients registered in our HBCR between January 1, 2014, and December 31, 2016, with the aim to know the pattern of cancer incidence in Bihar across the past 3 years.

Data reported regarding the incidence of cancer in eastern states are scarce. Nandi et al. reported 4484 patients registered from January 2005 to December 2006 where females outnumbered male by 1.3:1. Most common cancer among males was head-and-neck cancer while cervix and breast cancer were common among females. ${ }^{[6]}$ Another study by Ghosh et al. of 2058 patients over 3-year period demonstrated similar findings of head-and-neck cancer being most common followed by breast and cervical cancer. ${ }^{[7]}$ They also showed rising trend of gallbladder cancer in West Bengal. Similar finding was also reported by Maiti et al. who analyzed single largest center data for 5 years in West Bengal. ${ }^{[8]}$

Our study includes the largest $(n=6629)$ number of cancer patients reported from Bihar exploring the pattern and trends of cancer cases registered in HBCR. Unlike the above studies reported from neighboring states of Uttar Pradesh and West Bengal, our study finds gallbladder cancer as the most common cancer in Bihar overall. Head-and-neck cancer and breast cancer stand as the most common cancer among males and females, respectively. Unlike seen in major urban cancer registries in India where breast cancer is showing an increasing trend with fall in cervical cancer incidence, our data show both breast and cervical cancer having decreasing trend. ${ }^{[9]}$ Major concern is increasing projection of new cancer gallbladder cases detected yearly. The exact cause of which is still unknown but hypothesis including continuous arsenic exposure and proximity of river Ganga is postulated. ${ }^{[10]}$ Equally alarming is the rising incidence of head-and-neck cancer in Bihar despite ban on tobacco-related products by government. ${ }^{[1]]}$

The objective of HBCR is for the improvement in quality of care and dealing with administrative challenges unlike PBCRs where public health surveillance and epidemiological studies form higher goals. Our HBCR records every consecutive patient registered in Regional Cancer Centre, the diagnosis of which is required to be confirmed by histopathological examination including cytology or biopsy. Our hospital is a multispecialty hospital where other parallel specialties such as urology, gastrointestinal surgery, neurosurgery, pediatric surgery, and gynecology do see a substantial number of tumors which are

Table 1: List of ten most common cancers registered between January 2014 and December 2016 in descending orders in Hospital-Based Cancer Registry, Regional Cancer Centre, Patna, Bihar

\begin{tabular}{|c|c|c|c|c|c|c|c|}
\hline \multirow[t]{2}{*}{ Rank } & \multirow[t]{2}{*}{ Cancer } & \multicolumn{3}{|c|}{$n$} & \multirow[t]{2}{*}{ Total $(n)$} & \multirow{2}{*}{$\begin{array}{c}\text { Median } \\
\text { age (years) }\end{array}$} & \multirow[t]{2}{*}{ Male:female } \\
\hline & & 2014 & 2015 & 2016 & & & \\
\hline 1 & Gallbladder & 364 & 497 & 543 & 1404 & 55 & 0.60 \\
\hline 2 & Head and neck & 335 & 459 & 481 & 1275 & 53 & 1.8 \\
\hline 3 & Breast & 278 & 395 & 345 & 1018 & 46 & 0.02 \\
\hline 4 & Cervix & 230 & 279 & 204 & 713 & 50 & - \\
\hline 5 & Hematolymphoid & 127 & 139 & 152 & 418 & 33 & 1.3 \\
\hline 6 & Colon- rectum & 90 & 118 & 181 & 389 & 40 & 1.4 \\
\hline 7 & Stomach & 99 & 93 & 132 & 324 & 55 & 1.7 \\
\hline 8 & Ovary & 68 & 96 & 127 & 291 & 45 & - \\
\hline 9 & Esophagus & 42 & 54 & 74 & 170 & 60 & 2.0 \\
\hline 10 & Lung cancer & 40 & 49 & 68 & 157 & 55 & 1.5 \\
\hline
\end{tabular}


later confirmed as cancer on pathology and are managed by individual specialty. We do not claim to register all such cases as some of them may not be referred for cancer registry data entry. Moreover, sites where facility for biopsy are still suboptimal due paucity of infrastructure, manpower, and skills, especially for eloquent location such as lung mass, retroperitoneal sarcoma, and brain tumors may be under represented.

\section{Conclusion}

Gallbladder and head-and-neck cancer are the most common cancer seen in Bihar with both showing upward trend, whereas breast and cervical cancers are gradually decreasing. Government authorities need to invest more to provide sufficient manpower, infrastructure, and financial resources to tackle this rising cancer burden in Bihar. This also merits more stringent measures for ensuring tobacco ban and exploring methods to reduce chronic exposure of heavy metals.

\section{Financial support and sponsorship}

Nil.

\section{Conflicts of interest}

There are no conflicts of interest.

\section{References}

1. Available from: https://www.uicc.org/iarc-release- latest-world-cancer-statistics. [Last accessed on 2018 Sep 21].

2. Available from: http://www.gco.iarc.fr/today/ online-analysis-map?projection=globe. [Last accessed on 2018 Sep 21].

3. D'Souza ND, Murthy NS, Aras RY. Projection of cancer incident cases for India-till 2026. Asian Pac J Cancer Prev 2013;14:4379-86.

4. Available from: http://www.canceratlasindia.org/chapter 3 1. aspx. [Last accessed on 2018 Sep 21].

5. Available: http://www.censusindia.gov.in/Tables Published/A-Series/ A-Series_links/t_00_005.aspx. [Last accessed on 2018 Sep 21].

6. Nandi $\bar{M}$, Mandal $\bar{A}$, Asthana AK. Audit of cancer patients from Eastern Uttar Pradesh (UP), India: A university hospital based two year retrospective analysis. Asian Pac J Cancer Prev 2013;14:4993-8.

7. Ghosh N, Bandopadhyay R, Tirkey L, Das DK. Trend and pattern of various types of cancer with special reference to gall bladder cancer in North Bengal medical college, West Bengal, India: A 3 years record based study. Int J Prev Med 2015;6:24.

8. Maiti PK, Jana U, Ray A, Karmakar R, Mitra TN, Ganguly S, et al. Patterns of cancer occurrence in different regions of West Bengal - A hospital based study. J Indian Med Assoc 2012;110:445-8.

9. Asthana S, Chauhan S, Labani S. Breast and cervical cancer risk in India: An update. Indian J Public Health 2014;58:5-10.

10. Madhawi R, Pandey A, Raj S, Mandal M, Devi S, Sinha PK, et al. Geographical pattern of carcinoma gallbladder in Bihar and its association with river Ganges and arsenic levels: Retrospective individual consecutive patient data from regional cancer centre. South Asian J Cancer 2018;7: 167-70.

11. Available from: http://www.biharchamber.org/sites/default/files/ BiharGazette/D_Websites_eGazette_GazettePublished_408_2_2016. pdf. [Last accessed on 2018 Sep 21]. 